\title{
MCP-1 targeting: Shutting off an engine for tumor development (Review)
}

\author{
LIANG WANG $^{1^{*}}$, JINXIN LAN $^{2 *}$, JIAPING TANG $^{2,3^{*}}$ and NA LUO ${ }^{2,3}$ \\ ${ }^{1}$ Department of Urology, Tianjin Medical University General Hospital, Tianjin 300052; \\ ${ }^{2}$ Department of Anatomy and Histology, School of Medicine; ${ }^{3}$ Tianjin Key Laboratory of \\ Tumour Microenvironment and Neurovascular Regulation, Nankai University, Tianjin 300071, P.R. China
}

Received September 14, 2021; Accepted November 2, 2021

DOI: $10.3892 / \mathrm{ol} .2021 .13144$

\begin{abstract}
A large amount of research has proven that monocyte chemotactic protein-1 (MCP-1) is associated with different types of disease, including autoimmune, metabolic and cardiovascular diseases. In addition, several studies have found that MCP-1 is associated with tumor development. MCP-1 expression level in the tumor microenvironment is associated with tumor development, including in tumor invasion and metastasis, angiogenesis, and immune cell infiltration. However, the precise mechanism involved is currently being investigated. MCP-1 exerts its effects mainly via the MCP-1/C-C motif chemokine receptor 2 axis and leads to the activation of classical signaling pathways, such as PI3K/Akt/mTOR, ERK/GSK-3 $\beta /$ Snail, c-Raf/MEK/ERK and MAPK in different cells. The specific mechanism is still under debate; however, target therapy utilizing MCP-1 as a neutralizing antibody has been found to have a detrimental effect on tumor development. The aim of the present review was to examine the effect of MCP-1 on tumor development from several aspects, including its structure, its involvement in signaling pathways, the participating cells, and the therapeutic agents targeting MCP-1. The improved understanding into the structure of MCP-1 and the mechanism of action may facilitate new and practical therapeutic agents to achieve maximum performance in the treatment of patients with cancer.
\end{abstract}

\section{Contents}

1. Introduction

2. MCP-1 is a key protein in tumor development

3. MCP-1 is a potential target for tumor therapy

4. Conclusion

Correspondence to: Dr Na Luo, Department of Anatomy and Histology, School of Medicine, Nankai University, 94 Weijin Road, Tianjin 300071, P.R. China

E-mail: luon11@nankai.edu.cn

*Contributed equally

Key words: monocyte chemotactic protein-1, target therapy, tumor development

\section{Introduction}

Approximately 10 million patients succumb to various types of cancer annually, despite a wide variety of available cancer therapies. Malignant cancers demonstrate a poor prognosis, which is evidenced by a reduced expected lifespan and a greater difficulty in treatment. The advent of target therapies that suppress tumor growth, invasion and metastasis, has revolutionized cancer treatment and given optimism to numerous patients with cancer. In this regard, target therapy elicits both a specific and precise action on cancer cells, thereby reducing unpleasant side effects by contrast to traditional cancer treatment. Target therapy directed against lung cancer with the EGFR mutation has already demonstrated encouraging results $(1,2)$. Consequently, the enthusiasm for target therapy remains high since almost all types of cancer possess a key cellular factor that promotes its pathological biochemical metabolism.

The chemokine, monocyte chemoattractant protein-1 (MCP-1), also known as $\mathrm{C}-\mathrm{C}$ motif chemokine ligand 2 (CCL2), belongs to the C-C chemokine superfamily, which is comprised of at least 4 members (MCP-1, -2, -3 and -4). MCP-1 binds to a G-protein coupled receptor and plays a major role in the promotion of inflammation by modulating monocyte and basophil activity, but not neutrophil or eosinophil activity (3). Regardless of the affinities, MCP-1 has an ability to interact with a number of receptors [e.g., ACKR1, C-C motif chemokine receptor (CCR)-2, CCR5, CCR10 and CCR11] (4-8); however, previous findings suggested that CCR2 is the primary MCP-1 receptor. MCP-1 was initially identified in 1989 and termed glioma-derived chemotactic factor-2 (GDCF-2) (9). Later, GDCF-2 was found in the tissue culture media of phytohemagglutinin-stimulated human mononuclear leukocytes. With amino acid sequencing and cloning, GDCF-2 was finally renamed MCP-1 (10,11). MCP-1 is also known as tumor-derived chemotactic factor, as a wide variety of tumor cells can produce it (12). In addition, MCP-1 is secreted by a range of cell types in the tumor microenvironment (TME), such as fibroblasts, tumor-infiltrating monocytes, endothelial cells and tumor-associated adipocytes $(13,14)$.

The MCP-1 gene (SCYA2) is located on human chromosome 17q11.2-q21.1 (15). The precursor MCP-1 comprises 99 amino acids, with 23 amino acids at the $\mathrm{N}$-terminal, as the hydrophobic signal peptide, whereas the mature protein is comprised of 
76 amino acids, after cleavage of the signal peptide (Fig. 1A). There are two forms of the MCP-1 structure, known as I and $\mathrm{P}$ (Fig. 1B and C) (16). For all the MCPs, the N-terminal residues, 1-6, are essential for chemoattractant activity, and the first amino acid is necessary for direct receptor binding (17). Handel and Domaille (18) reported that the secondary structure of MCP- 1 consists of one $\alpha$-helix and four $\beta$-sheets (the grey label), including residues 9-11 ( $\beta 0), 27-31$ ( $\beta 1), 40-45$ ( $\beta 2$ ) and 51-54 ( $\beta 3$ ), which are different from the data in the Protein Data Bank (18). The latter shows that MCP-1 has three $\alpha$-helices. Residue 14 can be glycosylated, which can slightly decrease the potency of its chemotactic activity (19).

MCP-1 has been associated with several diseases, such as HIV-1 pathogenesis, cardiovascular disease and cancer. In the present review, the role and mechanism of MCP-1 in cancer are to be discussed.

\section{MCP-1 is a key protein in tumor development}

Cancer cell heterogeneity within a tumor is well-established due to the acquired mutations, as a result of the selective pressure caused by cell proliferation. Some of these acquired mutations result in the synthesis of cytokines that either activate or deactivate signaling pathways, allowing the cancer cell to escape leukocyte attack or to proliferate faster, leading to a higher survival probability for cancer cells. Consequently, MCP-1, secreted by the cancer cells, results in an advantage for the tumor but a disadvantage for the host, despite the specific signaling pathway involved. For example, MCP-1 expression is induced by IL- $1 \beta$ and regulated by NF- $\kappa B$ and activator protein-1 (AP-1) in renal cell carcinoma and glioblastoma $(20,21)$. MCP-1 is also a downstream molecule of IL-33, which increases tumor metastasis and invasion in esophageal carcinoma cells (22). MCP-1 can also be mediated by the mTOR complex 1 signaling pathway or sushi domain containing 2 in tumor cells (23). A long non-coding RNA LINC01296, termed lymph node metastasis associated transcript 1 , activates MCP-1 expression by interacting with hnRNPL and mediating H3K4 trimethylation (24). MCP-1 expression is also mediated by PA28 $\gamma$, which promotes tumor migration, invasion and angiogenesis (25). In addition, MCP-1 expression is mediated by angiotensin II binding to the angiotensin type 2 receptor and IL-4 in endothelial cells $(26,27)$. Furthermore, TGF- $\beta$ signaling has been associated with MCP-1 expression in fibroblast cells (28). The aforementioned findings indicate that MCP- 1 could be activated by different signaling pathways and contribute to tumor progression (Fig. 2).

MCP-1 and CCR2 are expressed in numerous types of cancer cells $(29,30)$. However, MCP-1 expression may vary in different cancer cell lines that originate from the same organ. For example, MCP-1 has a higher expression level in invasive breast cancer cell lines (e.g., BT594, Hs578T and MDA-MB-231) compared to non-invasive breast cancer cell lines (e.g., MCF7 and T47D) (31).

MCP-1 secretion by cancer cells portends to a poor clinical outcome, due to the induction of both tumor-associated macrophage infiltration and tumor metastasis in several solid tumors [e.g., non-small cell lung cancer (NSCLC), prostate cancer, breast cancer, ovarian cancer, and hepatocellular carcinoma] (32-36). MCP-1 secretion by Schwann cells also portends to a poor clinical outcome, due to the induction of perineural invasion (i.e., the local extension of cancer along nerves) (37). The abnormal stimulation of MCP-1 can be treated with drugs (e.g., minocycline, telmisartan and zoledronic acid), which have been reported to affect glioblastoma stromal cells (38).

Previous findings have demonstrated that the TME has been associated with tumorigenesis due to direct or indirect interaction between surrounding cells (i.e., stromal cells, fibroblasts, endothelial cells, and innate and adaptive immune cells) and tumor cells. The indirect interaction could build a two-way bridge via various cytokines, chemokines, and other factors, including MCP-1 $(13,14)$.

MCP-1 has been associated with tumor development in various manners. For example, i) MCP-1 recognizes and binds directly to CCR2-expressing cancer cells, which encourages tumor growth and invasiveness; ii) MCP-1 recruits monocytes into the tumor, which then differentiate into tumor-associated macrophages (TAMs) encouraging tumor development and angiogenesis; iii) MCP-1 acts directly on endothelial cells to produce endothelial growth factors, which encourages angiogenesis (39); and iv) MCP-1 recruits fibrocytes into the TME and enhances the formation of stroma (40) (Fig. 3). In summary, MCP-1 stimulates activation of various signaling pathways, which promotes tumor growth on the one hand. On the other hand, MCP-1 causes immune-suppression, which encourages tumor growth indirectly. Furthermore, MCP-1 enhances resistance to tumor drugs. MCP-1 expression increases resistance to an antiangiogenic agent, while the MCP-1 inhibitor (mNOX-E36), a L-RAN oligonucleotide chain, restores the sensitivity to the antiangiogenic agent (41).

MCP-1 has been studied mainly in cancer with a high incidence rate (e.g., breast, prostate and lung cancers). In this regard, $24.5 \%$ of all new cancer cases in women are due to breast cancer, while $14.1 \%$ of all new cancer cases in men are due to prostate cancer, and $11.4 \%$ of all new cancer cases in both men and women are due to lung cancer according to the International Agency for Research on Cancer (42). MCP-1 is associated with tumor development in a multi-faceted process and we hypothesized that this multi-faceted process may be analogous in various types of cancer with a high incidence rate. The multi-faceted process of MCP-1 association with tumor development will be subsequently discussed.

MCP-1 acts on cancer cells directly. The MCP-1/CCR2 signaling pathway may operate in an autocrine manner to promote tumor development, as some cancer cells secrete MCP-1 and express CCR 2 simultaneously. Previous studies (39-41) have addressed specific mechanisms by which MCP-1 has been associated with tumor development.

MCP-1 elevates IL-6 and TNF- $\alpha$, whose downstream oncogenic signaling pathways involve STAT3 and NF- $\kappa B$ in a hepatocellular carcinoma mouse model. Furthermore, treatment with an MCP-1 specific antibody (Ab) blocks the oncogene, c-MYC, which is downstream of STAT3 and thereby reduces tumor proliferation in a hepatocellular carcinoma mouse model (36). The aforementioned findings suggest that MCP-1 plays a key role in the activation of oncogenes and promotion of tumorigenesis. 

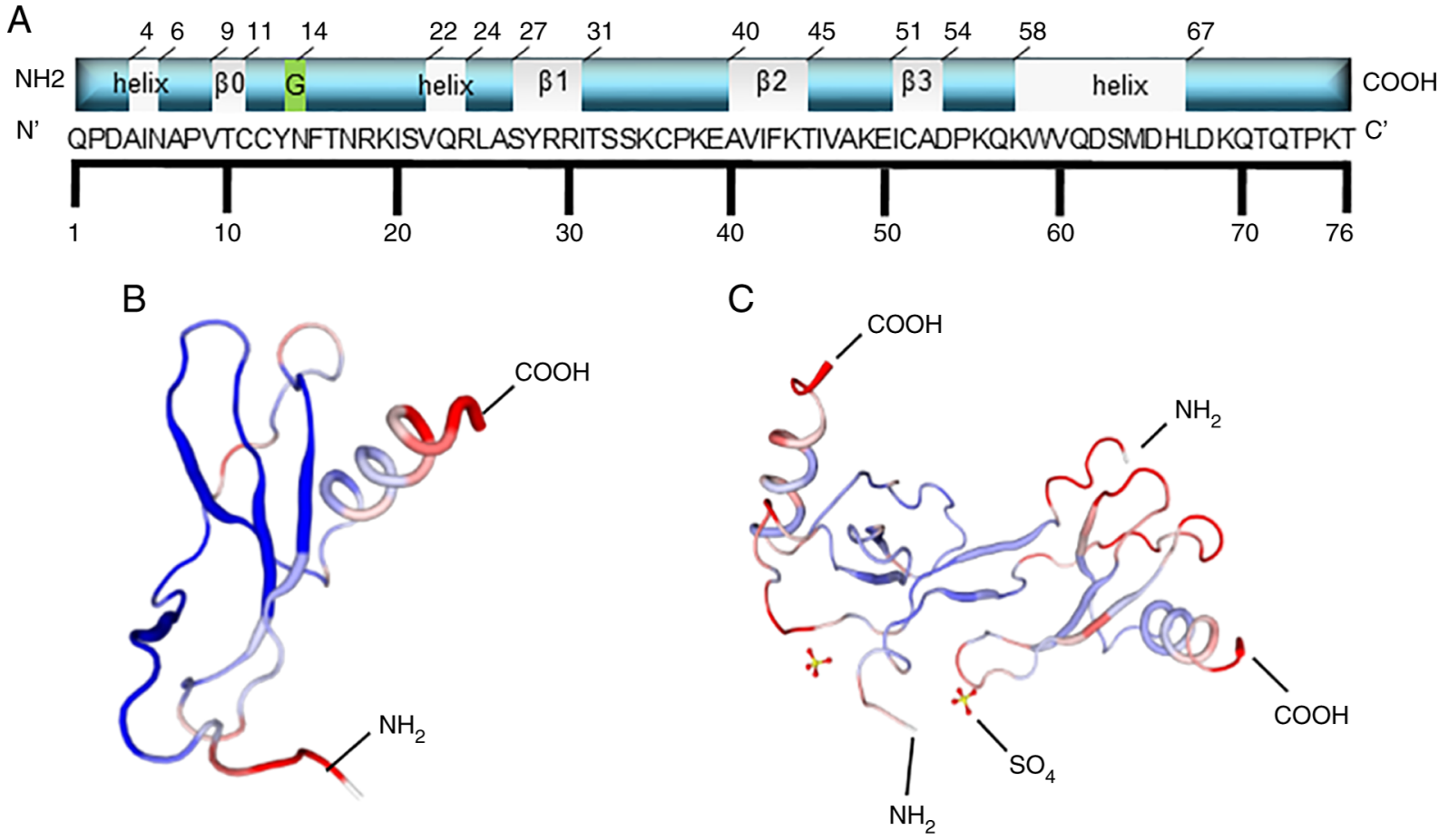

Figure 1. Schematic structural illustration of MCP1. (A) The schematic structural illustration. For all MCPs, N-terminal residues 1-6 are essential for chemoattractant activity, and the first amino acid is necessary for direct receptor binding. MCP-1 is composed of 76 amino acids, and the secondary structure of MCP-1 consisted of one $\alpha$-helix and four regions of $\beta$-sheet (the grey label), including residues 9-11 ( $\beta 0$ ), residues 27-31 ( $\beta 1$ ), residues $40-45$ ( $\beta 2$ ), residues 51-54 ( $\beta 3$ ), which is little different from the data in PDB protein bank. The last one shows that MCP-1 has three $\alpha$-helix (the grey sections). Residue 14 can be glycosylated (the green section), which can slightly decrease the potency of the chemotactic activity of MCP-1. (B and C) The two forms of secondary structures of MCP-1: (B) is form I and (C) is form P. The former is the single MCP-1 molecule, while the latter is the dimer.

In addition, adipocyte secretion of MCP-1, in the TME, binds to CCR 2 on cancer cells and activates the PI3K/Akt/mTOR signaling pathway. The activation of the $\mathrm{PI} 3 \mathrm{~K} / \mathrm{Akt} / \mathrm{mTOR}$ signaling pathway induces hypoxia inducible factor-1 $\alpha$, which mediates vascular endothelial growth factor (VEGF)-A expression and thereby stimulates tumor angiogenesis $(35,43)$. The activation of the PI3K/Akt/mTOR signaling pathway by MCP-1 also inhibits autophagy and stimulates tumor proliferation in prostate cancer cells and osteosarcoma cells $(30,44,45)$. MCP-1 also induces Akt activation in a dose-dependent manner (46). In addition to the activation of the PI3K/Akt/mTOR signaling pathway, the c-Raf/MEK/ERK and MAPK signaling pathways play a role in MCP-1-induced tumor migration (47). Furthermore, the IP3-dependent Akt/PKB signaling pathway is associated with MCP-1-induced tumor proliferation and migration (48).

MCP-1 can also induce MMP production in cancer cells and thereby promotes tumor progression (49). MCP-1 enhances the aggressiveness of NSCLC cells by increasing the level of MMP-9 expression in vitro (50). MCP-1 induces MMP production by activating the ERK $1 / 2$ and $\mathrm{p} 38$ MAPK signaling pathways, and upregulates MMP by activating c-Raf/Raf-1, MEK, ERK, MAPK, c-Jun, NF- $\kappa B$ and AP-1 $(47,51,52)$. MMP cleaves cell-to-cell and cell-extracellular matrix adhesion components, which promotes cell detachment and leads to epithelial-mesenchymal transition (EMT) and enhances metastasis (53). In addition, MCP-1 can directly induce EMT by activating the ERK/GSK-3//Snail signaling pathway (54). Overall, MCP-1 stimulates tumor proliferation and metastasis by activating the MAPK/ERK and ERK1/2-MMP2/9 signaling pathways, respectively $(48,55)$.

In addition, previous studies $(30,35,44-46)$ have found that MCP-1 specifically recognizes the CCR2 receptor and induces a series of signaling pathways that alter cancer cell metabolism. The mechanism involved shows consistency among various tumor types in which MCP1 activates classic signaling pathways, even though the exact mechanism involved remains unclear. Thus, the aforementioned findings suggest that MCP-1 may be a novel target for cancer therapy.

MCP-1 facilitates endothelial cell angiogenesis. Excessive angiogenesis is a salient feature of various tumors, which produces a highly unorganized and permeable tumor vasculature compared with that in normal cells. The leaky neo-capillaries within the tumor, not only provide less oxygen/nutrients to the tumor, but also form an abnormal TME promoting tumor development. The endothelial cells are the protagonist during the multi-step process of angiogenesis, and previous studies indicate that MCP-1 interacts with endothelial cells and may therefore be associated with angiogenesis in tumor development $(56,57)$.

MCP-1 downregulates the expression level of TNF superfamily-15 (TNFSF15), which is an inhibitor of neovascularization (58). In addition, elevated MCP-1 expression levels were positively correlated with VEGF expression levels, a potent angiogenic factor (39). MCP-1 also regulates the interaction between cancer cells and endothelial cells in vitro, and promotes endothelial cell migration, thereby promoting angiogenesis (57). The binding of MCP-1 to CCR2 activates 


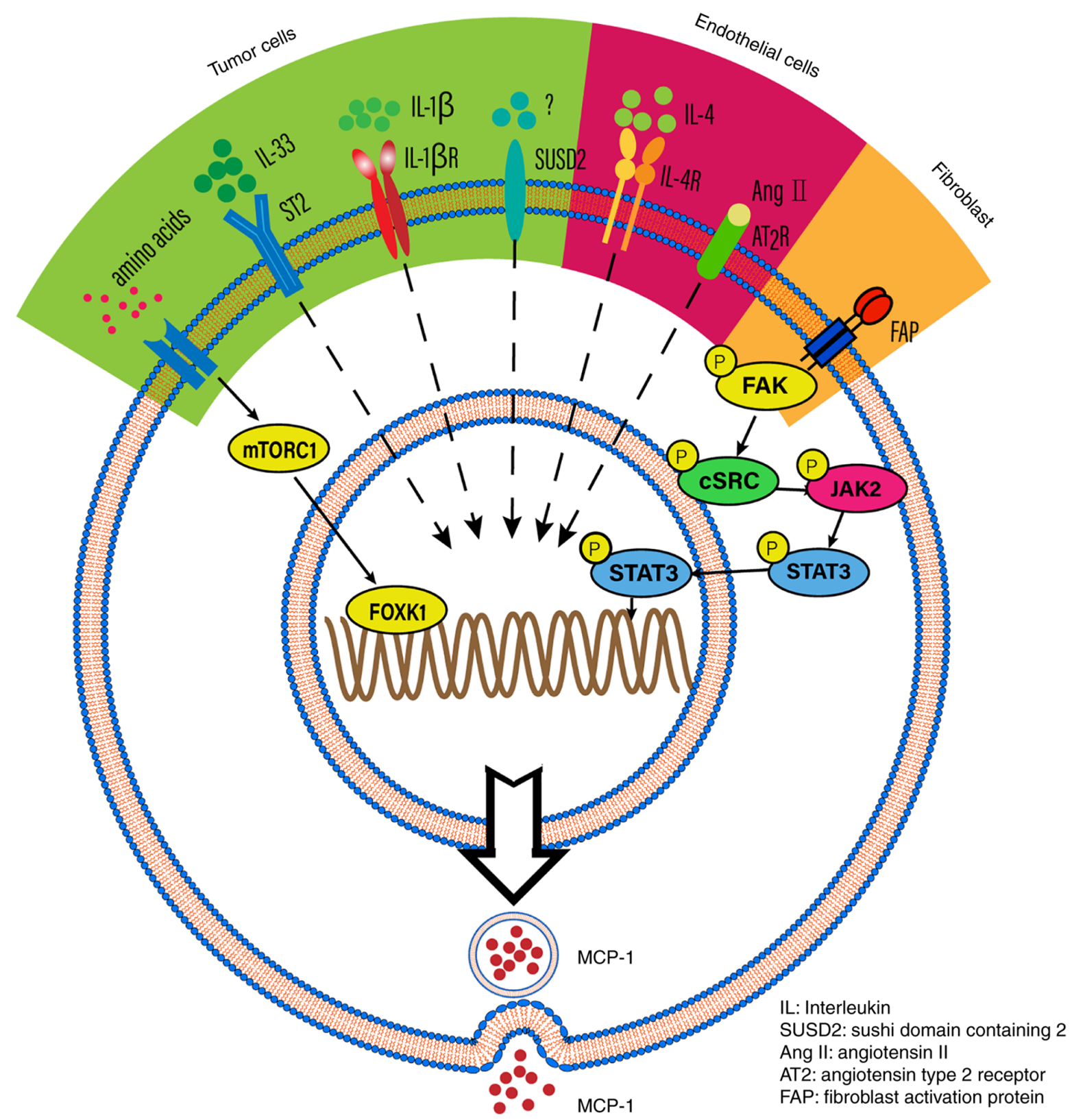

Figure 2. The potential mechanisms that regulate MCP-1 expression. MCP-1 expression is induced by IL-1 $\beta$ in specific tumor cells. MCP-1 is downstream of IL-33, thus IL-33 which binds to its receptor (ST2) may impact on MCP-1 secretion. MCP-1 is also mediated by mTORC1, which is stimulated by amino acids . IN addition, MCP-1 expression is also promoted by SUSD2 in tumor cells. Concerning endothelial cells, angiotensin II and IL-4 can mediate the expression of MCP-1. Fibroblast activation protein can promote MCP-1 expression in cancer-associated fibroblasts. FAP, fibroblast activation protein; IL, interleukin; SUSD2, sushi domain containing 2; AngII, angiotensin II; AT2, angiotensin type 2 receptor; FAP, fibroblast activation protein.

the PI3K/Akt signaling pathway and induces phosphorylation of p38, ERK1/2, Src in endothelial cells in vitro (59).

MCP-1 promotes monocyte/macrophage recruitment in the $T M E$. In addition to endothelial cells, MCP-1 is also associated with regulating the immune microenvironment in the tumor. Myeloid-derived suppressor cells (MDSCs), as a major regulator of immune responses in cancer, bearing the markers CD11b (CR3A or integrin $\alpha \mathrm{M}$ ) and Gr-1 [anti-Gr-1 monoclonal (m)Abs recognize epitopes common to Ly6C and Ly6G] (60), infiltrate the tumor tissue under hypoxia, oxidative agents, pro-inflammatory cytokines or nutrient scarcity $(39,61)$. Monocytic MDSCs are derived from circulating Ly6 $\mathrm{C}^{\text {hi }}$ monocytes, originate from either a myeloid or splenic reservoir in a CCR2-dependent manner, and acquire a pro-inflammatory signature that affects lymphocyte activity, proliferation and survival $(62,63)$. In addition, fibroblast activation protein-induced cancer-associated fibroblasts promote the recruitment of MDSCs via the production of MCP-1 (64). Furthermore, MDSC differentiation into TAMs represents one of the major immune cells in the TME in most types of cancer. Blocking the MCP-1/CCR2 axis leads to a notable decrease in TAM abundance (65). TAMs remodel the TME, which promotes EMT and angiogenesis (65), and are divided into two categories, the antitumor M1-like and pro-tumor M2-like TAMs. MCP-1 increases the number of M2 TAMs but decreases the number of M1 TAMs. This also promotes TAM-dependent lymphangiogenesis in bladder cancer (24), 


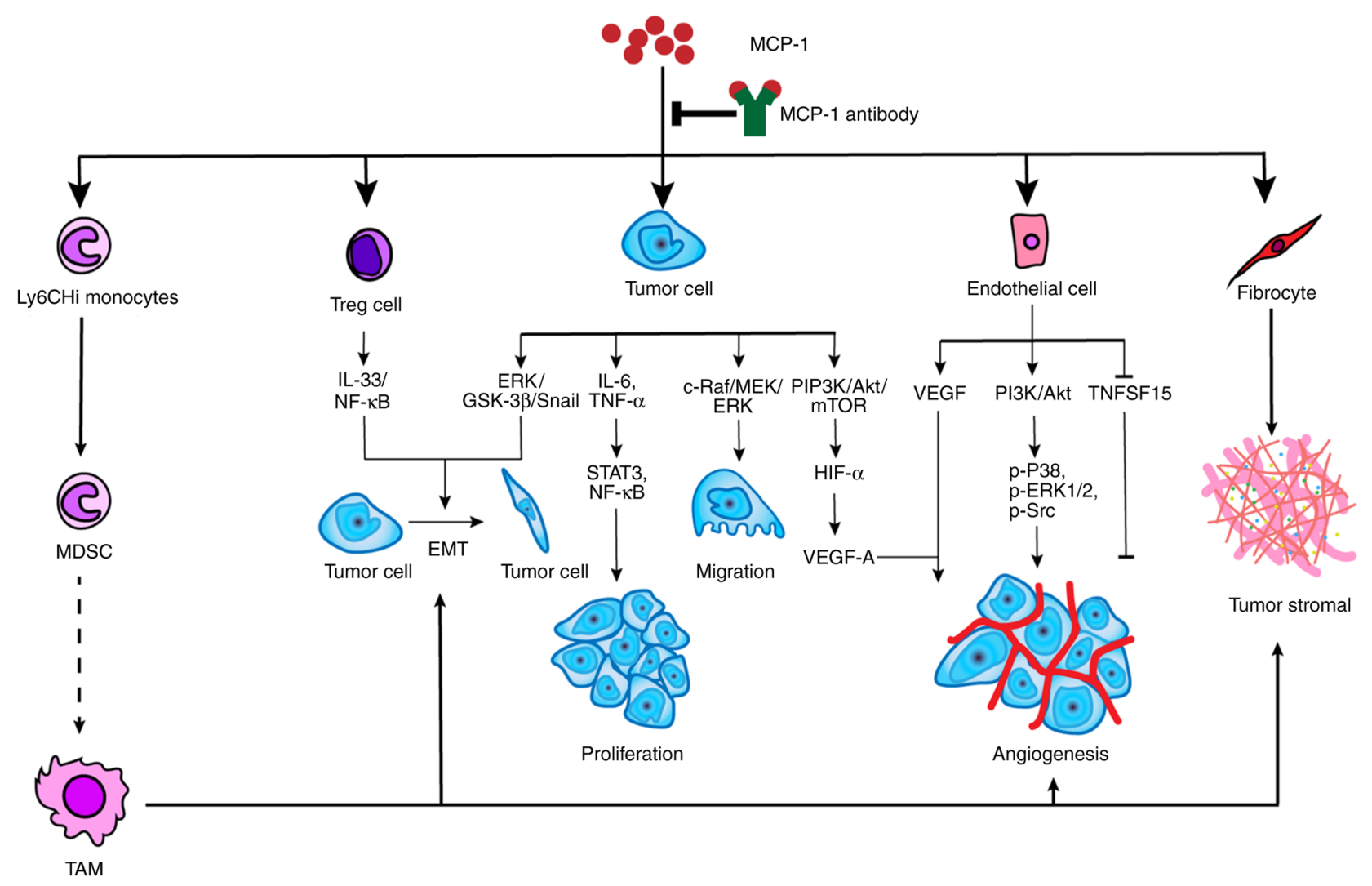

Figure 3. The potential mechanism of MCP-1-promoted tumor development. MCP-1 can not only utilize monocytes, Treg cells, endothelial cells and fibrocytes but also act on tumor directly to affect tumor development. The precise mechanism of MDSC transforming into TAM is not clear. TAM advances tumor development in many respects, including EMT, angiogenesis and tumor stromal formation. Treg cells and tumor cells can induce the process of EMT through IL-33/NF- $\mathrm{kB}$ and ERK/GSK-3 $\alpha /$ Snail, respectively. MCP-1 facilitates tumor proliferation, migration and angiogenesis through specific pathways, such as ERK/GSK-3 $\beta /$ Snail for migration, and PI3K/Akt/mTOR for angiogenesis. In addition, MCP-1 also promotes tumor angiogenesis by acting on endothelial cells via the PI3K/Akt signaling pathway and suppressing TNFSF15. MCP-1 is also a promoter for fibrocytes that synthesize tumor stromal. Notably, MCP-1 is the engine for tumor development and shutting off the engine will inhibit this process. TNFS15, tumor necrosis factor superfamily-15; TAM, tumor-associated macrophage; MDSC, myeloid-derived suppressor cell; MCP-1, monocyte chemotactic protein-1; EMT, epithelial-mesenchymal transition; Treg, regulatory T lymphocyte.

which results in the immune escape of tumor cells, initiation of blood vessel growth, and finally the metastasis of tumor cells. A CCR2 antagonist reduces the number of M2 TAMs and production of cytokines (i.e., IL-6, CCL2, KC, G-CSF, MIP-1 and MIP-2), which enhances the efficacy of tumor therapies (66).

Notably, MCP-1 assists in the recruitment of monocytes and their differentiation into macrophages, which suggests that MCP-1 is a key target molecule in tumor development. Furthermore, MCP-1 modulates the progression of mammary tumorigenesis, primarily due to its ability to recruit macrophages to the TME. The loss of MCP-1 expression results in a decline of macrophage markers, and reduces primary tumor volume and delays tumor progression in a triple negative breast cancer model (67).

MCP-1 expression and TAM recruitment demonstrate a positive correlation, while inhibition of MCP-1 activity reduces monocyte infiltration, TAM accumulation and tumor incidence (61). Activation of the MCP-1/CCR2 axis promotes the recruitment of monocytes and TAMs into the TME in several tumor types, including sarcoma and breast cancer (68). Monocyte recruitment into the tumor metastatic site occurs in an MCP-1-dependent manner, and their transformation into macrophages promotes tumor proliferation, metastatic tumor survival/growth, and a poor prognosis in various types of cancer [e.g., breast, prostate, bladder, kidney and NSCLC) $(34,41,69)$. Knockdown of 5'-nucleotidase domain containing 2 notably reduces TAM recruitment via suppression of the MCP-1/CCR2 signaling pathway in colorectal carcinoma (70). Furthermore, as aforementioned, Tgfbr $2^{\mathrm{FspKO}}$ improves the level of MCP-1 secretion and enhances tumor progression associated with TAM recruitment, which indicates the MCP-1-dependent attraction of macrophages into the TME depends upon the effects of the surrounding cytokines. By contrast, recent findings showed that MCP-1 recruits and activates macrophages to kill cancer cells in various types of cancer (e.g., gastric and colorectal cancer, and melanoma), and MCP-1 expression was decreased in small cell lung cancer (69).

MCP-1 regulates monocyte attraction and infiltration by the induction of adhesive molecules and cytokines, along with binding to the CCR 2 receptor on monocytes. The signaling pathway of MCP-1-induced monocyte/TAM recruitment is not clear, but it has been reported that the JAK/STAT and p42/44 MAPK/c-Jun pathways may be involved in the activation of macrophages $(71,72)$. Furthermore, macrophage infiltration promotes angiogenesis with MCP-1 expression. Elevated MCP-1 expression levels promote both macrophage infiltration and angiogenesis (73). The number of newly formed vascular tubes significantly increases when MCP-1-expressing cancer cells interact with macrophages compared with 
Table I. MCP-1 neutralizing antibodies currently available.

\begin{tabular}{|c|c|c|c|c|c|}
\hline Name & Type & Company name & Time & Application (Refs.) & Remarks (Refs.) \\
\hline $2 \mathrm{H} 5$ & Mouse MCP-1 antibody & $\begin{array}{l}\text { eBioscience/ } \\
\text { BD Biosciences }\end{array}$ & 1994 & $\begin{array}{l}\text { Umbilical cord Mesenchymal } \\
\text { stem cells (84) }\end{array}$ & $\begin{array}{l}\text { Cross-reacted with } \\
\text { human MCP-1 (85) }\end{array}$ \\
\hline 5D3-F7 & $\begin{array}{l}\text { Recombinant human } \\
\text { MCP-1 antibody }\end{array}$ & BD Biosciences & 1994 & Human sarcoma (86) & \\
\hline AF-479-NA & Mouse MCP-1 antibody & R\&D systems & 2000 & $\begin{array}{l}\text { Human gastric cancer (87); } \\
\text { breast cancer ( } 88)\end{array}$ & $\begin{array}{l}\text { Cross-reacted with } \\
\text { human MCP-1 (87) }\end{array}$ \\
\hline MAB479 & Mouse MCP-1 antibody & R\&D systems & 2003 & Mouse lung cancer (89) & \\
\hline MAB679 & Human MCP-1 antibody & R\&D systems & 2004 & $\begin{array}{l}\text { Human clear cell renal cell } \\
\text { carcinoma (90) }\end{array}$ & \\
\hline MAB279 & $\begin{array}{l}\text { Human MCP-1 } \\
\text { biotinylated antibody }\end{array}$ & R\&D systems & 2004 & $\begin{array}{l}\text { Human glioblastoma } \\
\text { multiforme (91); human breast } \\
\text { cancer cell line MCF10CA1d } \\
\text { (CA1d) (78) }\end{array}$ & \\
\hline AF-279-NA & Human MCP-1 antibody & R\&D systems & 2004 & Human lung cancer (92) & \\
\hline $\begin{array}{l}\text { CNTO888/ } \\
\text { carlumab }\end{array}$ & Human MCP-1 antibody & Centocor Inc. & 2007 & Human prostate cancer (33) & \\
\hline C1142 & Mouse MCP-1 antibody & Centocor Inc. & 2007 & Prostate cancer (93) & \\
\hline
\end{tabular}

MCP-1-expressing cancer cells only (41), indicating that MCP-1 promotes angiogenesis via macrophage attraction.

MCP-1 recruits regulatory Tlymphocytes (Tregs) into the TME. In addition to monocytes/macrophages in the TME, MCP-1 also affects the activity of Tregs. MCP-1 recruits Treg lymphocytes into the TME via the IL-33/NF- $\kappa \mathrm{B}$ signaling pathway, which promotes tumor development, whereas, Treg lymphocyte recruitment into the TME fails to occur in the absence of MCP-1 (22,74). MCP-1 also recruits Treg lymphocytes into the TME via the downregulation of TNFSF15, whereby TNFSF15 levels are inversely correlated with the degree of $\mathrm{CD}^{+}{ }^{+} \mathrm{CD} 25^{+} \mathrm{FOXP} 3^{+}$Treg lymphocyte infiltration (58). In this regard, there is a reduction of $\mathrm{CD}^{+}{ }^{+} \mathrm{FOXP} 3^{+}$Treg lymphocytes and induction of $\mathrm{CD}^{+}$T-lymphocyte cytotoxicity, which restricts tumor growth in a CCR2 knockout mouse lung adenocarcinoma model (75). Similarly, blocking of the MCP-1/CCR4 signaling pathway using a CCR4 antagonist inhibits tumor growth and prolongs survival time in patients with head and neck squamous cell carcinoma (HNSCC) (76). The aforementioned findings indicate that MCP-1 recruits Treg lymphocytes into the TME and reduces the antitumor responses of effector $\mathrm{T}$ lymphocytes by binding to $\mathrm{MCP}-1$ receptors (Fig. 3).

\section{MCP-1 is a potential target for tumor therapy}

MCP-1 directly or indirectly mediates changes in the tumor, which promotes tumor progression and metastasis. This suggests that blocking the effects of MCP-1 may serve as a novel anticancer therapeutic strategy. MCP-1 target therapy is divided into two categories, MCP-1 inhibitor and MCP-1 neutralizing $\mathrm{Ab}$. The latter is described, as it has a prospective clinical application (Table I).

Previous studies have indicated that MCP-1 overexpression occurs in various cancer cells $(34,66)$. The blocking of the MCP-1/CCR2 signaling pathway inhibits tumor progression $(29,46)$ and weakens recruitment of M2 macrophages and Tregs, which activates antitumor $\mathrm{CD}^{+} \mathrm{T}$ lymphocytes $(32,66)$. MCP-1 neutralizing Ab (CNTO888) treatment delays tumor growth in an in vivo xenograft mouse model of prostate cancer (33) and inhibits the dissemination of estrogen-dependent breast cancer induced by macrophages in a zebrafish model (77). MCP-1 target therapy inhibits the development of hepatocellular carcinoma by blocking the oncogenic IL-6 and TNF- $\alpha$ signaling pathways and activating NK cells in the TME (36). Treatment with anti-MCP-1 mAb does not change the total leukocyte recruitment, but does change neutrophil and M2 macrophage recruitment (32). However, MCP-1 neutralizing $\mathrm{Ab}$ treatment inhibits cancer cell proliferation in vitro, but not in vivo (78). The reason for this difference may be due to the fact that the MCP-1 neutralizing mAbs cannot be delivered to the TME in vivo at an effective concentration for drug efficacy.

In addition, MCP-1 target therapy and other antitumor therapy can play a synergistic effect. Anti-MCP-1 administration enhances the effect of cisplatin by suppressing colony formation in HNSCC in vitro (79), and MCP-1 inhibitor (mNOX-E36) therapy enhances bevacizumab inhibition in tumor progression (41).

Furthermore, since the N-terminal but not C-terminal is essential for all MCP signaling and chemotactic activity, this domain may be a potential target. As early as 1999, Van Coillie identified the N-terminal truncated MCP-2 (the 6th amino acid serine to 76th amino acid proline) can block the activity of intact MCP-1 (80), as the homology sequences between MCP-1 and MCP-2 is $62 \%(17,80)$.

\section{Conclusion}

MCP-1 acts as an engine that drives tumor progression and therefore may serve as an effective therapeutic target. In this 
regard, the CCR2 blockade (i.e., the MCP-1 receptor) shows promise due to the antitumor effects it exerts $(29,36,66,75)$. However, MCP-1 is secreted not only by tumor cells, but also by stromal cells surrounding the tumor parenchyma (81). MCP-1 neutralizing Ab treatment may be more advantageous as a clinical strategy than CCR2 blockade for three main reasons as indicated below.

First, MCP-1 binds to other CC-chemokine receptors (besides CCR2), which promote tumorigenesis. Therefore, MCP-1 neutralizing Ab treatment may block not only the tumorigenic effects of CCR2 binding, but also the tumorigenic effects of the other CC-chemokine receptor binding. Second, MCP-1 neutralizing Ab treatment reduces MCP-1 serum levels, which decreases systemic inflammation and contributes to a favorable prognosis (82). Third, drug development based on MCP-1 neutralizing Ab treatment appears to have more promise in delivering a highly efficient therapeutic treatment.

However, the use of MCP-1 neutralizing Ab requires further research. Traditionally, it has been argued that cessation of anti-MCP-1 treatment led to a rebound of MCP-1 and a notable increase in metastases (83). It points out the issue of utilizing the MCP-1 neutralizing Ab correctly, which will improve the side effects. Antitumor drugs may be selected according to tumor types, and observe the principle of concomitant drugs and full course of treatment. Certainly, it is on the premise of the development of an ideal antibody of MCP-1.

In conclusion, further research is required to reveal the role of MCP-1 in cancer progression in order to identify the most beneficial target and design the most effective therapeutic strategy.

\section{Acknowledgements}

Not applicable.

\section{Funding}

Funding was obtained from the General Project of Applied Basic and Cutting-edge Technology, Tianjin Science and Technology Commission (grant no. 20JCYBJC01500), and the National Natural Science Foundation of China (grant nos. 81872325 and 82172640 ).

\section{Availability of data and materials}

Not applicable.

\section{Authors' contributions}

LW was the major person in charge of the organizing and writing of the revised manuscript during the peer review process. JL searched the associated papers, interpreted the information and wrote the draft. JT searched the associated papers, interpreted the information and wrote the outline of the draft. NL was in charge of writing, interpreting and overseeing the manuscript. All authors read and approved the final manuscript. Data authentication is not applicable.

\section{Ethics approval and consent to participate}

Not applicable.

\section{Patient consent for publication}

Not applicable.

\section{Competing interests}

The authors declare that they have no competing interests.

\section{References}

1. da Cunha Santos G, Shepherd FA and Tsao MS: EGFR mutations and lung cancer. Annu Rev Pathol 6: 49-69, 2011.

2. Paez JG, Jänne PA, Lee JC, Tracy S, Greulich H, Gabriel S, Herman P, Kaye FJ, Lindeman N, Boggon TJ, et al: EGFR mutations in lung cancer: Correlation with clinical response to gefitinib therapy. Science 304: 1497-1500, 2004.

3. Deshmane SL, Kremlev S, Amini S and Sawaya BE: Monocyte chemoattractant protein-1 (MCP-1): An overview. J Interferon Cytokine Res 29: 313-326, 2009.

4. Blanpain CD, Migeotte I, Lee B, Vakili J, Doranz BJ, Govaerts C, Vassart G, Doms RW and Parmentier M: CCR5 binds multiple CC-chemokines: MCP-3 acts as a natural antagonist. Blood 94: 1899-1905, 1999.

5. Bonini JA, Martin SK, Dralyuk F, Roe MW, Philipson LH and Steiner DF: Cloning, expression, and chromosomal mapping of a novel human CC-chemokine receptor (CCR10) that displays high-affinity binding for MCP-1 and MCP-3. DNA Cell Biol 16: 1249-1256, 1997.

6. Hemmerich S, Paavola C, Bloom A, Bhakta S, Freedman R, Grunberger D, Krstenansky J,Lee S, McCarley D, Mulkins M, et al: Identification of residues in the monocyte chemotactic protein-1 that contact the MCP-1 receptor, CCR2. Biochemistry 38: 13013-13025, 1999.

7. Kashiwazaki M, Tanaka T, Kanda H, Ebisuno Y, Izawa D, Fukuma N, Akimitsu N, Sekimizu K, Monden M and Miyasaka M: A high endothelial venule-expressing promiscuous chemokine receptor DARC can bind inflammatory, but not lymphoid, chemokines and is dispensable for lymphocyte homing under physiological conditions. Int Immunol 15: 1219-1227, 2003.

8. Schweickart VL, Epp A, Raport CJ and Gray PW: CCR11 is a functional receptor for the monocyte chemoattractant protein family of chemokines. J Biol Chem 275: 9550-9556, 2000.

9. Robinson EA, Yoshimura T, Leonard EJ, Tanaka S, Griffin PR, Shabanowitz J, Hunt DF and Appella E: Complete amino acid sequence of a human monocyte chemoattractant, a putative mediator of cellular immune reactions. Proc Natl Acad Sci USA 86: 1850-1854, 1989.

10. Yoshimura T, Robinson EA, Tanaka S, Appella E and Leonard EJ: Purification and amino acid analysis of two human monocyte chemoattractants produced by phytohemagglutinin-stimulated human blood mononuclear leukocytes. J Immunol 142: 1956-1962, 1989.

11. Yoshimura T, Yuhki N, Moore SK, Appella E, Lerman MI and Leonard EJ: Human monocyte chemoattractant protein-1 (MCP-1). Full-length cDNA cloning, expression in mitogen-stimulated blood mononuclear leukocytes, and sequence similarity to mouse competence gene JE. FEBS Lett 244: 487-493, 1989.

12. Bottazzi B, Colotta F, Sica A, Nobili N and Mantovani A: A chemoattractant expressed in human sarcoma cells (tumor-derived chemotactic factor, TDCF) is identical to monocyte chemoattractant protein-1/monocyte chemotactic and activating factor (MCP-1/MCAF). Int J Cancer 45: 795-797, 1990.

13. Fujisaki K, Fujimoto H, Sangai T, Nagashima T, Sakakibara M, Shiina N, Kuroda M, Aoyagi Y and Miyazaki M: Cancer-mediated adipose reversion promotes cancer cell migration via IL- 6 and MCP-1. Breast Cancer Res Treat 150: 255-263, 2015.

14. Wu Q, Li B, Li Z, Li J, Sun S and Sun S: Cancer-associated adipocytes: Key players in breast cancer progression. J Hematol Oncol 12: 95, 2019.

15. Mehrabian M, Sparkes RS, Mohandas T, Fogelman AM and Lusis AJ: Localization of monocyte chemotactic protein-1 gene (SCYA2) to human chromosome 17q11.2-q21.1. Genomics 9: 200-203, 1991

16. Lubkowski J, Bujacz G, Boqué L, Domaille PJ, Handel TM and Wlodawer A: The structure of MCP-1 in two crystal forms provides a rare example of variable quaternary interactions. Nat Struct Biol 4: 64-69, 1997. 
17. Zhang Y, Ernst CA and Rollins BJ: MCP-1: Structure/activity analysis. Methods 10: 93-103, 1996.

18. Handel TM and Domaille PJ: Heteronuclear $(1 \mathrm{H}, 13 \mathrm{C}, 15 \mathrm{~N})$ NMR assignments and solution structure of the monocyte chemoattractant protein-1 (MCP-1) dimer. Biochemistry 35: 6569-6584, 1996.

19. Proost P, Struyf S, Couvreur M, Lenaerts JP, Conings R, Menten P, Verhaert P, Wuyts A and Damme JV: Posttranslational modifications affect the activity of the human monocyte chemotactic proteins MCP-1 and MCP-2: Identification of MCP-2(6-76) as a natural chemokine inhibitor. J Immunol 160 4034-4041, 1998.

20. Jung Y, Ahn SH, Park H, Park SH, Choi K, Choi C, Kang JL and Choi YH: MCP-1 and MIP-3 $\alpha$ secreted from necrotic cell-treated glioblastoma cells promote migration/infiltration of microglia. Cell Physiol Biochem 48: 1332-1346, 2018.

21. Lee CH, Hung PF, Lu SC, Chung HL, Chiang SL, Wu CT, Chou WC and Sun CY: MCP-1/MCPIP-1 signaling modulates the effects of IL-1 $\beta$ in renal cell carcinoma through ER stress-mediated apoptosis. Int J Mol Sci 20: 6101, 2019.

22. Yue Y, Lian J, Wang T, Luo C, Yuan Y, Qin G, Zhang B and Zhang Y: Interleukin-33-nuclear factor- $\mathrm{BB}-\mathrm{CCL} 2$ signaling pathway promotes progression of esophageal squamous cell carcinoma by directing regulatory T cells. Cancer Sci 111: 795-806, 2020.

23. Nakatsumi H, Matsumoto M and Nakayama KI: Noncanonical pathway for regulation of CCL2 expression by an mTORC1FOXK1 axis promotes recruitment of tumor-associated macrophages. Cell Rep 21: 2471-2486, 2017.

24. Chen C, He W, Huang J, Wang B, Li H, Cai Q, Su F, Bi J, Liu H, Zhang B, et al: LNMAT1 promotes lymphatic metastasis of bladder cancer via CCL2 dependent macrophage recruitment. Nat Commun 9: 3826, 2018.

25. Liu S, Liu D, Zeng X, Wang J, Liu J, Cheng J, Lei K, Bai H, Ji N, Zhou M, et al: PA28 $\gamma$ acts as a dual regulator of IL-6 and CCL2 and contributes to tumor angiogenesis in oral squamous cell carcinoma. Cancer Lett 428: 192-200, 2018.

26. Castiñeiras-Landeira MI, Rodiño-Janeiro BK, ParadelaDobarro B, Batista-Oliveira AL, Raposeiras-Roubín S, GonzálezPeteiro M, González-Juanatey JR and Álvarez E: Change of concept about the regulation of angiotensin II-induced monocyte chemoattractant protein-1 production in human endothelial cells. Vascul Pharmacol 80: 20-34, 2016.

27. Rollins BJ and Pober JS: Interleukin-4 induces the synthesis and secretion of MCP-1/JE by human endothelial cells. Am J Pathol 138: 1315-1319, 1991.

28. Hembruff SL, Jokar I, Yang L and Cheng N: Loss of transforming growth factor-beta signaling in mammary fibroblasts enhances CCL2 secretion to promote mammary tumor progression through macrophage-dependent and -independent mechanisms. Neoplasia 12: 425-433, 2010.

29. Kuper C, Beck FX and Neuhofer W: Autocrine MCP-1/CCR2 signaling stimulates proliferation and migration of renal carcinoma cells. Oncol Lett 12: 2201-2209, 2016.

30. Lu Y, Cai Z, Galson DL, Xiao G, Liu Y, George DE, Melhem MF, Yao Z and Zhang J: Monocyte chemotactic protein-1 (MCP-1) acts as a paracrine and autocrine factor for prostate cancer growth and invasion. Prostate 66: 1311-1318, 2006.

31. Mohamed HT, El-Ghonaimy EA, El-Shinawi M, Hosney M, Götte M, Woodward WA, El-Mamlouk T and Mohamed MM: IL-8 and MCP-1/CCL2 regulate proteolytic activity in triple negative inflammatory breast cancer a mechanism that might be modulated by Src and Erk1/2. Toxicol Appl Pharmacol 401: $115092,2020$.

32. Fridlender ZG, Kapoor V, Buchlis G, Cheng G, Sun J, Wang LC, Singhal S, Snyder LA and Albelda SM: Monocyte chemoattractant protein-1 blockade inhibits lung cancer tumor growth by altering macrophage phenotype and activating CD8+ cells. Am J Respir Cell Mol Biol 44: 230-237, 2011.

33. Loberg RD, Ying C, Craig M, Yan L, Snyder LA and Pienta KJ: CCL2 as an important mediator of prostate cancer growth in vivo through the regulation of macrophage infiltration. Neoplasia 9: 556-562, 2007.

34. Qian BZ, Li J, Zhang H, Kitamura T, Zhang J, Campion LR, Kaiser EA, Snyder LA and Pollard JW: CCL2 recruits inflammatory monocytes to facilitate breast-tumour metastasis. Nature 475: 222-225, 2011.

35. Sun C, Li X, Guo E, Li N, Zhou B, Lu H, Huang J, Xia M, Shan W, Wang B, et al: MCP-1/CCR-2 axis in adipocytes and cancer cell respectively facilitates ovarian cancer peritoneal metastasis. Oncogene 39: 1681-1695, 2020.
36. Teng KY, Han J, Zhang X, Hsu SH, He S, Wani NA, Barajas JM, Snyder LA, Frankel WL, Caligiuri MA, et al: Blocking the CCL2-CCR2 ax is using CCL2-neutralizing antibody is an effective therapy for hepatocellular cancer in a mouse model. Mol Cancer Ther 16: 312-322, 2017.

37. Bakst RL, Xiong H, Chen CH, Deborde S, Lyubchik A, Zhou Y, He S, McNamara W, Lee SY, Olson OC, et al: Inflammatory monocytes promote perineural invasion via CCL2-mediated recruitment and cathepsin B expression. Cancer Res 77: 6400-6414, 2017.

38. Salacz M, Kast RE, Saki N, Brüning A, Karpel-Massler G and Halatsch ME: Toward a noncytotoxic glioblastoma therapy: Blocking MCP-1 with the MTZ regimen. Onco Targets Ther 27: 2535-2545, 2016

39. Ueno T, Toi M, Saji H, Muta M, Bando H, Kuroi K, Koike M, Inadera $\mathrm{H}$ and Matsushima K: Significance of macrophage chemoattractant protein-1 in macrophage recruitment, angiogenesis, and survival in human breast cancer. Clin Cancer Res 6: 3282-3289, 2000

40. Kuziel G, Thompson V,D'Amato JV and ArendtLM: Stromal CCL2 signaling promotes mammary tumor fibrosis through recruitment of myeloid-lineage cells. Cancers (Basel) 12: 2083, 2020.

41. Cho HR, Kumari N, Vu HT, Kim H, Park CK and Choi SH: Increased antiangiogenic effect by blocking CCL2-dependent macrophages in a rodent glioblastoma model: Correlation study with dynamic susceptibility contrast perfusion MRI. Sci Rep 9: $11085,2019$.

42. Sung H, Ferlay J, Siegel RL, Laversanne M, Soerjomataram I, Jemal A and Bray F: Global cancer statistics 2020: GLOBOCAN estimates of incidence and mortality worldwide for 36 cancers in 185 countries. CA Cancer J Clin 71: 209-249, 2021.

43. Guru SK, Pathania AS, Kumar S, Ramesh D, Kumar M, Rana S, Kumar A, Malik F, Sharma PR, Chandan BK, et al: Secalonic acid-D represses HIF1alpha/VEGF-mediated angiogenesis by regulating the $\mathrm{Akt} / \mathrm{mTOR} / \mathrm{p} 70 \mathrm{~S} 6 \mathrm{~K}$ signaling cascade. Cancer Res 75: 2886-2896, 2015.

44. Zhang J, Lu Y and Pienta KJ: Multiple roles of chemokine (C-C motif) ligand 2 in promoting prostate cancer growth. J Natl Cancer Inst 102: 522-528, 2010.

45. Chen Q, Sun W, Liao Y, Zeng H, Shan L, Yin F, Wang Z, Zhou Z, Hua Y and Cai Z: Monocyte chemotactic protein-1 promotes the proliferation and invasion of osteosarcoma cells and upregulates the expression of AKT. Mol Med Rep 12: 219-225, 2015.

46. Loberg RD, Day LL, Harwood J, Ying C, John LN, Giles R, Neeley CK and Pienta KJ: CCL2 is a potent regulator of prostate cancer cell migration and proliferation. Neoplasia 8: 578-586, 2006.

47. Liu JF, Chen PC, Chang TM and Hou CH: Monocyte chemoattractant protein-1 promotes cancer cell migration via c-Raf/MAPK/AP-1 pathway and MMP-9 production in osteosarcoma. J Exp Clin Cancer Res 39: 254, 2020.

48. He S and Zhang X: The rs1024611 in the CCL2 gene and risk of gynecological cancer in Asians: A meta-analysis. World J Surg Oncol 16: 34, 2018.

49. Ito $\mathrm{Y}$, Ishiguro $\mathrm{H}$, Kobayashi $\mathrm{N}$, Hasumi $\mathrm{H}$, Watanabe M, Yao M and Uemura $\mathrm{H}$ : Adipocyte-derived monocyte chemotactic protein-1 (MCP-1) promotes prostate cancer progression through the induction of MMP-2 activity. Prostate 75: 1009-1019, 2015.

50. An J, Xue Y, Long M, Zhang G, Zhang J and Su H: Targeting CCR2 with its antagonist suppresses viability, motility and invasion by downregulating MMP-9 expression in non-small cell lung cancer cells. Oncotarget 8: 39230-39240, 2017.

51. Tang CH and Tsai CC: CCL2 increases MMP-9 expression and cell motility in human chondrosarcoma cells via the Ras/Raf/MEK/ERK/NF- $\kappa$ B signaling pathway. Biochem Pharmacol 83: 335-344, 2012.

52. Yang CQ, Li W, Li SQ, Li J, Li YW, Kong SX, Liu RM, Wang SM and Lv WM: MCP-1 stimulates MMP-9 expression via ERK 1/2 and p38 MAPK signaling pathways in human aortic smooth muscle cells. Cell Physiol Biochem 34: 266-276, 2014.

53. Orlichenko LS and Radisky DC: Matrix metalloproteinases stimulate epithelial-mesenchymal transition during tumor development. Clin Exp Metastasis 25: 593-600, 2008.

54. Li S, Lu J, Chen Y, Xiong N, Li L, Zhang J, Yang H, Wu C, Zeng $\mathrm{H}$ and Liu Y: MCP-1-induced ERK/GSK-3 $\beta /$ snail signaling facilitates the epithelial-mesenchymal transition and promotes the migration of MCF-7 human breast carcinoma cells. Cell Mol Immunol 14: 621-630, 2017.

55. Liu W, Wang L, Zhang J, Qiao L, Liu Y, Yang X, Zhang J, Zheng W and Ma Z: Purification of recombinant human chemokine CCL2 in $E$. coli and its function in ovarian cancer. 3 Biotech 11: 8, 2021. 
56. Salcedo R, Ponce ML, Young HA, Wasserman K, Ward JM, Kleinman HK, Oppenheim JJ and Murphy WJ: Human endothelial cells express CCR2 and respond to MCP-1: Direct role of MCP-1 in angiogenesis and tumor progression. Blood 96: 34-40, 2000.

57. Wang S, Xu M, Li F, Wang X, Bower KA, Frank JA, Lu Y, Chen G, Zhang Z, Ke Z, et al: Ethanol promotes mammary tumor growth and angiogenesis: The involvement of chemoattractant factor MCP-1. Breast Cancer Res Treat 133: 1037-1048, 2012.

58. Deng W, Gu X, Lu Y, Gu C, Zheng Y, Zhang Z, Chen L, Yao Z and Li LY: Down-modulation of TNFSF15 in ovarian cancer by VEGF and MCP-1 is a pre-requisite for tumor neovascularization. Angiogenesis 15: 71-85, 2012.

59. Arefieva TI, Kukhtina NB, Antonova OA and Krasnikova TL: MCP-1-stimulated chemotaxis of monocytic and endothelial cells is dependent on activation of different signaling cascades. Cytokine 31: 439-446, 2005.

60. Bronte V, Brandau S, Chen SH, Colombo MP, Frey AB, Greten TF Mandruzzato S, Murray PJ, Ochoa A, Ostrand-Rosenberg S, et al: Recommendations for myeloid-derived suppressor cell nomenclature and characterization standards. Nat Commun 7: 12150, 2016.

61. Yang H, Zhang Q, Xu M, Wang L, Chen X, Feng Y, Li Y, Zhang X, Cui W and Jia X: CCL2-CCR2 axis recruits tumor associated macrophages to induce immune evasion through PD-1 signaling in esophageal carcinogenesis. Mol Cancer 19: 41, 2020

62. Guilliams M, Mildner A and Yona S: Developmental and functional heterogeneity of monocytes. Immunity 49: 595-613, 2018.

63. Shand FH, Ueha S, Otsuji M, Koid SS, Shichino S Tsukui T, Kosugi-Kanaya M, Abe J, Tomura M, Ziogas J and Matsushima K: Tracking of intertissue migration reveals the origins of tumor-infiltrating monocytes. Proc Natl Acad Sci USA 111: 7771-7776, 2014.

64. Yang X, Lin Y, Shi Y, Li B, Liu W, Yin W, Dang Y, Chu Y, Fan J and He R: FAP promotes immunosuppression by cancer-associated fibroblasts in the tumor microenvironment via STAT3-CCL2 signaling. Cancer Res 76: 4124-4135, 2016.

65. Laviron $\mathrm{M}$ and Boissonnas A: Ontogeny of tumor-associated macrophages. Front Immunol 10: 1799, 2019.

66. Li X, Yao W, Yuan Y, Chen P, Li B, Li J, Chu R, Song H, Xie D, Jiang $\mathrm{X}$, et al: Targeting of tumour-infiltrating macrophages via CCL2/CCR2 signalling as a therapeutic strategy against hepatocellular carcinoma. Gut 66: 157-167, 2017.

67. Cranford TL, Velázquez KT, Enos RT, Bader JE, Carson MS, Chatzistamou L, Nagarkatti M and Murphy EA: Loss of monocyte chemoattractant protein-1 expression delays mammary tumorigenesis and reduces localized inflammation in the C3(1)/SV40Tag triple negative breast cancer model. Cancer Biol Ther 18: 85-93, 2017.

68. Li F, Kitajima S, Kohno S, Yoshida A, Tange S, Sasaki S, Okada N, Nishimoto Y, Muranaka H, Nagatani N, et al: Retinoblastoma inactivation induces a protumoral microenvironment via enhanced CCL2 secretion. Cancer Res 79: 3903-3915, 2019.

69. Zheng Y, Wang Z, Wei S, Liu Z and Chen G: Epigenetic silencing of chemokine CCL2 represses macrophage infiltration to potentiate tumor development in small cell lung cancer. Cancer Lett 499: 148-163, 2021.

70. Zhu Z, Hou Q and Guo H: NT5DC2 knockdown inhibits colorectal carcinoma progression by repressing metastasis, angiogenesis and tumor-associated macrophage recruitment: A mechanism involving VEGF signaling. Exp Cell Res 397: 112311, 2020.

71. Sodhi A and Biswas SK: Monocyte chemoattractant protein-1induced activation of p42/44 MAPK and c-Jun in murine peritoneal macrophages: A potential pathway for macrophage activation. J Interferon Cytokine Res 22: 517-526, 2002

72. Biswas SK and Sodhi A: Tyrosine phosphorylation-mediated signal transduction in MCP-1-induced macrophage activation: Role for receptor dimerization, focal adhesion protein complex and JAK/STAT pathway. Int Immunopharmacol 2: 1095-1107, 2002

73. Kuroda T, Kitadai Y, Tanaka S, Yang X, Mukaida N, Yoshihara M and Chayama K: Monocyte chemoattractant protein-1 transfection induces angiogenesis and tumorigenesis of gastric carcinoma in nude mice via macrophage recruitment. Clin Cancer Res 11: 7629-7636, 2005.

74. Chang AL, Miska J, Wainwright DA, Dey M, Rivetta CV, Yu D, Kanojia D, Pituch KC, Qiao J, Pytel P, et al: CCL2 produced by the glioma microenvironment is essential for the recruitment of regulatory $\mathrm{T}$ cells and myeloid-derived suppressor cells. Cancer Res 76: 5671-5682, 2016
75. Mittal P, Wang L, Akimova T, Leach CA, Clemente JC, Sender MR, Chen Y, Turunen BJ and Hancock WW: The CCR2/MCP-1 chemokine pathway and lung adenocarcinoma. Cancers (Basel) 12: 3723, 2020.

76. Sun W, Li WJ, Wei FQ, Wong TS, Lei WB, Zhu XL, Li J and Wen WP: Blockade of MCP-1/CCR4 signaling-induced recruitment of activated regulatory cells evokes an antitumor immune response in head and neck squamous cell carcinoma. Oncotarget 7: 37714-37727, 2016.

77. Svensson S, Abrahamsson A, Rodriguez GV, Olsson AK, Jensen L, Cao Y and Dabrosin C: CCL2 and CCL5 are novel therapeutic targets for estrogen-dependent breast cancer. Clin Cancer Res 21: 3794-3805, 2015.

78. Yao M, Smart C, Hu Q and Cheng N: Continuous delivery of neutralizing antibodies elevate CCL2 levels in mice bearing MCF10CA1d breast tumor xenografts. Transl Oncol 10: 734-743, 2017.

79. Wichmann G, Körner C, Boehm A, Mozet C and Dietz A: Stimulation by monocyte chemoattractant protein-1 modulates the ex-vivo colony formation by head and neck squamous cell carcinoma cells. Anticancer Res 35: 3917-3924, 2015.

80. Van Coillie E, Van Damme J and Opdenakker G: The $\mathrm{MCP} / \mathrm{eotaxin}$ subfamily of CC chemokines. Cytokine Growth Factor Rev 10: 61-86, 1999.

81. Yoshimura T: The production of monocyte chemoattractant protein-1 (MCP-1)/CCL2 in tumor microenvironments. Cytokine 98: 71-78, 2017.

82. Laird BJA, Fallon M, Hjermstad MJ, Tuck S, Kaasa S, Klepstad P and McMillan DC: Quality of life in patients with advanced cancer: Differential association with performance status and systemic inflammatory response. J Clin Oncol 34: 2769-2775, 2016.

83. Bonapace L, Coissieux MM, Wyckoff J, Mertz KD, Varga Z, Junt T and Bentires-Alj M: Cessation of CCL2 inhibition accelerates breast cancer metastasis by promoting angiogenesis. Nature 515: 130-133, 2014.

84. Shen C, Lie P, Miao T, Yu M, Lu Q, Feng T, Li J, Zu T, Liu X and $\mathrm{Li} \mathrm{H}$ : Conditioned medium from umbilical cord mesenchymal stem cells induces migration and angiogenesis. Mol Med Rep 12: 20-30, 2015.

85. Luo Y,Laning J,Hayashi M,Hancock PR, Rollins B and Dorf ME: Serologic analysis of the mouse beta chemokine JE/monocyte chemoattractant protein-1. J Immunol 153: 3708-3716, 1994.

86. Peri G, Milanese C, Matteucci C, Ruco L, Zhou D, Sozzani S, Coletta I and Mantovani A: A new monoclonal antibody (5D3-F7) which recognizes human monocyte-chemotactic protein-1 but not related chemokines. Development of a sandwich ELISA and in situ detection of producing cells. J Immunol Methods 174: 249-257, 1994

87. Zhao C, Bu X, Wang W, Ma T and Ma H: GEC-derived SFRP5 inhibits Wnt5a-induced macrophage chemotaxis and activation. PLoS One 9: e85058, 2014.

88. Fujimoto H, Sangai T, Ishii G, Ikehara A, Nagashima T, Miyazaki M and Ochiai A: Stromal MCP-1 in mammary tumors induces tumor-associated macrophage infiltration and contributes to tumor progression. Int J Cancer 125: 1276-1284, 2009.

89. Roy RM, Wuthrich M and Klein BS: Chitin elicits CCL2 from airway epithelial cells and induces CCR2-dependent innate allergic inflammation in the lung. J Immunol 189: 2545-2552, 2012 .

90. Arakaki R, Yamasaki T, Kanno T, Shibasaki N, Sakamoto H, Utsunomiya N, Sumiyoshi T, Shibuya S, Tsuruyama T, Nakamura E, et al: CCL2 as a potential therapeutic target for clear cell renal cell carcinoma. Cancer Med 5: 2920-2933, 2016.

91. Lai SW, Liu YS, Lu DY and Tsai CF: Melatonin modulates the microenvironment of glioblastoma multiforme by targeting sirtuin 1. Nutrients 11: 1343, 2019.

92. Zhan Z, Xie X, Cao H, Zhou X, Zhang XD, Fan H and Liu Z: Autophagy facilitates TLR4- and TLR3-triggered migration and invasion of lung cancer cells through the promotion of TRAF6 ubiquitination. Autophagy 10: 257-268, 2014.

93. Loberg RD, Ying C, Craig M, Day LL, Sargent E, Neeley C, Wojno K, Snyder LA, Yan L and Pienta KJ: Targeting CCL2 with systemic delivery of neutralizing antibodies induces prostate cancer tumor regression in vivo. Cancer Res 67: 9417-9424, 2007.

This work is licensed under a Creative Commons Attribution-NonCommercial-NoDerivatives 4.0 International (CC BY-NC-ND 4.0) License. 